\title{
Length adaptation of smooth muscle contractile filaments in response to sustained activation
}

\author{
Jonas Stålhand and Gerhard A. Holzapfel
}

\section{Linköping University Post Print}

\section{Tweet}

N.B.: When citing this work, cite the original article.

Original Publication:

Jonas Stålhand and Gerhard A. Holzapfel, Length adaptation of smooth muscle contractile filaments in response to sustained activation, 2016, Journal of Theoretical Biology, (397), 1321.

http://dx.doi.org/10.1016/j.jtbi.2016.02.028

Copyright: Elsevier

http://www.elsevier.com/

Postprint available at: Linköping University Electronic Press

http://urn.kb.se/resolve?urn=urn:nbn:se:liu:diva-128721 


\title{
Length adaptation of smooth muscle contractile filaments in response to sustained activation
}

\author{
Jonas Stålhand ${ }^{1}$ And Gerhard A. Holzapfel ${ }^{2}$ \\ ${ }^{1}$ Solid Mechanics, Department of Management and Engineering, \\ Linköping University, Linköping, Sweden \\ ${ }^{2}$ Graz University of Technology, Institute of Biomechanics, Graz, \\ Austria
}

Appeared in

Journal of Theoretical Biology, 397, 2016, pp. 13-21

\begin{abstract}
Airway and bladder smooth muscles are known to undergo length adaptation under sustained contraction. This adaptation process entails a remodelling of the intracellular actin and myosin filaments which shifts the peak of the active force-length curve towards the current length. Smooth muscles are therefore able to generate the maximum force over a wide range of lengths. In contrast, length adaptation of vascular smooth muscle has attracted very little attention and only a handful of studies have been reported. Although their results are conflicting on the existence of a length adaptation process in vascular smooth muscle, it seems that, at least, peripheral arteries and arterioles undergo such adaptation. This is of interest since peripheral vessels are responsible for pressure regulation, and a length adaptation will affect the function of the cardiovascular system. It has, e.g., been suggested that the inward remodelling of resistance vessels associated with hypertension disorders may be related to smooth muscle adaptation. In this study we develop a continuum mechanical model for vascular smooth muscle length adaptation by assuming that the muscle cells remodel the actomyosin network such that the peak of the active stress-stretch curve is shifted towards the operating point. The model is specialised to hamster cheek pouch arterioles and the simulated response to stepwise length changes under contraction. The results show that the model is able to recover the salient features of length adaptation reported in the literature.
\end{abstract}




\section{Introduction}

Smooth muscle share many features with striated muscle. For example, the active force originates from a cyclic interaction between actin and myosin filaments and is dependent on the muscle length in a bell-shaped manner. In other aspects smooth muscle differs significantly from striated muscle. One of the most obvious differences is that smooth muscle lacks the highly regular organisation of contractile filaments which give rise to the banded structure in striated muscle. In addition, various types of smooth muscles are able to generate the same maximum isometric force over a wide range of lengths given time to adapt (Pratusevich et al., 1995; Kuo et al., 2003; Herrera et al., 2005; Martinez-Lemus et al., 2008; Tuna et al., 2011). This is in sharp contrast to striated muscle where the maximum isometric force is associated with a particular length (Gordon et al., 1966).

Evidence suggests that smooth muscle length adaptation is connected to a structurally and 'plastic-like' mechanism of contractile and other cytoskeletal filaments (Kuo et al., 2003; Herrera et al., 2005; Seow, 2005; van den Akker et al., 2010). Both actin and myosin polymerise and depolymerise rapidly in response to activation (Mehta \& Gunst, 1999; Herrera et al., 2002). This evanescence facilitates reorganisation of the contractile filaments in order to maintain a maximal overlap and, thereby, the maximal isometric force. The underlying mechanism for length adaptation is not well understood, but the predominant theory is that sustained activation promotes serial addition or subtraction of contractile filaments. This theory is also supported by experimental observations. First, the maximal isometric force remains invariant of the adapted length (Kuo et al., 2003; Herrera et al., 2005; Bossé et al., 2008). Second, the contraction velocity and power output are proportional to the adapted length (Kuo et al., 2003; Herrera et al., 2005). Third, the adenosine-triphosphate (ATP) consumption is also proportional to the adapted length (Kuo et al., 2003). Fourth, the myosin filament density in the smooth muscle cell increases linearly with the adapted length (Kuo et al., 2003; Bossé et al., 2008). The serial model is also the simplest model which accounts for all these observations and is, therefore, chosen herein.

Compared to the airways or the bladder, surprisingly little is known about length adaptation in vascular smooth muscle (van den Akker et al., 2010; Tuna et al., 2011). The published studies mostly concern small arteries and arterioles (Bakker et al., 2004; Martinez-Lemus et al., 2004; Tuna et al., 2013a,b), but larger arteries have also been reported (Syyong et al., 2008; Murtada et al., 2015). Since small arteries and arterioles are responsible for pressure regulation, it is reasonable to expect that length adaptation in these vessels will play a role for the function of the arterial system. For example, Tuna et al. (2011) suggested that length adaptation can be related to inward remodelling of resistance vessels which is a hallmark of 
hypertensive disorders. Normal arteries operate at diameters just below the maximum isometric force and the cytoskeleton does not contribute to the passive properties (Tuna et al., 2011, Fig. 1). Under a sustained contraction at low distension, e.g., following an acute pressure increase, the smooth muscle cells undergo length adaptation through a reorganisation of the cytoskeleton. The adaptation shifts the diameter for the maximum isometric force towards the new contracted and smaller diameter. The length adaptation is much faster than the remodelling of the extracellular matrix. As a consequence, the reorganised cytoskeleton will now act as a brake and resist a full dilation of the passive vessel back to the original diameter (MartinezLemus et al., 2004). The length adaptation process could, therefore, explain the inward remodelling seen in hypertension, at least partially.

Previously, mathematical models have been used to study length adaptation in airway smooth muscles, see, e.g., Silveira et al. (2005), Ijpma et al. (2011) and Donovan (2013). Models for length adaptation in the vascular system are very rare, however, particularly models based on continuum mechanics. A notable exception is the recent study of Murtada et al. (2015). The continuum mechanical modelling is equipped with several challenges. First, the limited number of studies for length adaptation in vascular smooth muscles makes it difficult to find relevant data. Second, the available experimental data are often obtained or manipulated in a way that makes them inappropriate for continuum mechanical modelling. For example, in uniaxial extension tests of muscles it is customary to measure the optimal length for which the generated force is maximised. The muscle length is then normalised with respect to this value rather than the undeformed length which is customary in solid mechanics. Furthermore, if the study fails to give the specimen's undeformed geometry, it is virtually impossible to compute the stress and deformation quantities needed in the modelling. Given these limitations, any study involving the length adaptation in vascular smooth muscle will be subject to indirect evidence and qualitative comparisons to existing studies for other smooth muscles. This does not diminish the importance of studies, however. Given a solid model based on fundamental physical principles, parameter studies can tell us much about the behaviour of the underlying process. Parameter studies can also act as test guide for future experiments.

In this paper, we develop a novel micro-mechanically motivated continuum model for contractile filament length adaptation in smooth muscle. The model is an extension of previously published smooth muscle contraction models (Stålhand et al., 2008; Murtada et al., 2012; Sharifimajd \& Stålhand, 2014) and is derived from fundamental principles to assert a mechanically and thermodynamically consistent behaviour. The number of parameters are also kept to a minimum to facilitate their identification from standard experiments. The model is presented in Sect. 2 and it is then specialised to hamster cheek pouch arterioles in Sect. 3. Finally, it is used to simulate 
length adaptation in Sect. 4.

\section{Continuum model}

In this section, we present a continuum model for smooth muscle length adaptation under sustained contraction. The model is nonlinear and assumes a homogeneous deformation field.

The force in the smooth muscle cell is generated by the contractile units, like in all other muscle cells. Even though there is no immediate equivalence to the contractile unit in a striated muscle, it is commonly defined to comprise two adjacent dense bodies including the actin and myosin filaments spanning between them. The contractile unit is modelled by a spring in series with a friction clutch. The spring accounts for the elasticity in the actomyosin network while the clutch symbolises the contractile effect generated by an ensemble of cycling cross-bridges.

\subsection{Kinematics}

Let the reference length of the contractile unit be $L_{0}$ and assume that the whole cell in its reference state comprises $N_{0}$ contractile units in series, see Fig. 1. Assume that a contraction, or an extension, can be described by a relative sliding of the filaments $u_{\mathrm{fs}}$, followed by an elastic displacement of the actomyosin network $u_{\mathrm{e}}$. Note that deformations are taken to be positive in extension throughout the text. Furthermore, assume that the filament length is uniform and remains invariant in the adaptation process. Then, any adaptation of the cell length is necessarily associated with a change in the number of contractile units in series. Let $N$ be the number of contractile units in series in the cell after adaptation such that its length $l$ in a contracted state becomes

$$
l=N\left(L_{0}+u_{\mathrm{fs}}+u_{\mathrm{e}}\right) .
$$

Since the deformation is assumed to be homogeneous, the stretch $\lambda$ can be computed by dividing Eq. (1) with the referential cell length $N_{0} L_{0}$, resulting to

$$
\lambda=n\left(1+\varepsilon_{\mathrm{fs}}+\varepsilon_{\mathrm{e}}\right),
$$

where $n=N / N_{0}$ is introduced together with the strain quantities $\varepsilon_{\mathrm{fs}}=$ $u_{\mathrm{fs}} / L_{0}$ and $\varepsilon_{\mathrm{e}}=u_{\mathrm{e}} / L_{0}$. Finally, length adaptation operates on a time scale of minutes to hours (Pratusevich et al., 1995; Seow, 2000; Syyong et al., 2008) while maximum smooth muscle contraction can be achieved within seconds. This implies that $\dot{n} \approx 0$ compared to the strain rate so that

$$
\dot{\lambda}=n\left(\dot{\varepsilon}_{\mathrm{fs}}+\dot{\varepsilon}_{\mathrm{e}}\right),
$$


where the employed superposed dot designates the material time derivative.

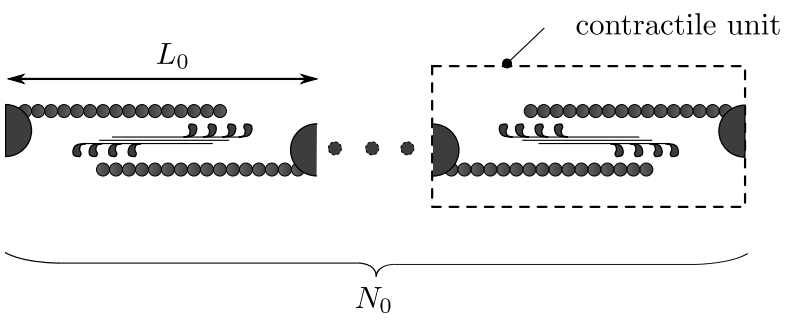

reference state

(before adaptation)

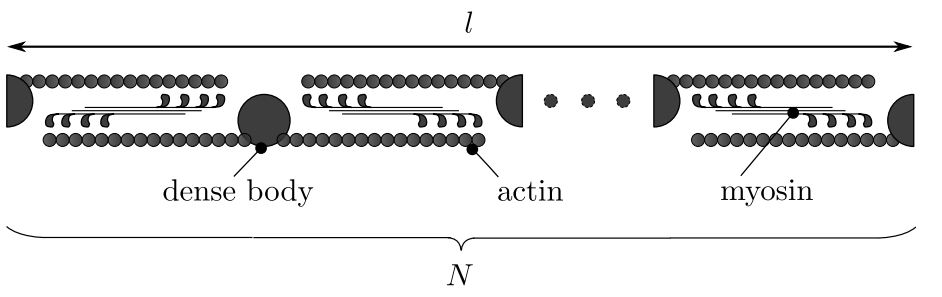

after adaptation

Figure 1: Schematic representation of the smooth muscle length adaptation. The reference state comprises $N_{0}$ contractile units in series which changes to $N$ contractile units after length adaptation.

Muscle contraction is associated with a microscopic interaction between filaments where myosin heads form cross-bridges to actin and translates the filament in the power stroke. After the translation is completed, crossbridges detach actin and the cycle starts again. Due to stochastic variations in the activation of myosin, there are periods when the fraction of attached myosin heads is reduced. During these periods, the external load can partly reverse the filament translation and momentarily elongate the muscle. This causes a microscopic jerking motion which is observed in experiments (Debold et al., 2005). On the macroscopic scale, the jerking motion is smoothed and the net effect is instead experienced as a slippage between cycling myosin heads and actin. This is consistent with the kinematics of a friction clutch (Sharifimajd \& Stålhand, 2013, 2014), and we use the clutch as a symbolic model for the force generated by an ensemble of cycling myosin. To that end, let the clutch rotate an angle $\theta$ such that it generates a contraction, see Fig. 2. The friction force exerted by the clutch is dependent on the slip speed between the clutch and the actin filament. Introduce the normalised slip speed $w$, defined by

$$
w=v+\dot{\varepsilon}_{\mathrm{fs}},
$$

where $v=r \dot{\theta} / L_{0}$ is the normalised clutch velocity and $r$ is the clutch radius. Note that the terms are added since $\dot{\varepsilon}_{\mathrm{fd}}$ is defined positive in extension. 


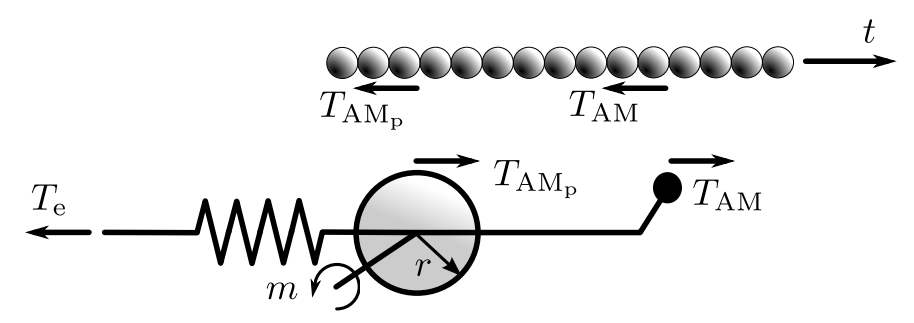

Figure 2: Free body diagram of the contracting unit. The total force $t$ and the thermodynamic force $m$ driving the friction clutch are external forces which are balanced by the internal forces $T_{\mathrm{AM}_{\mathrm{p}}}$ and $T_{\mathrm{AM}}$ associated with cycling and non-cycling cross-bridges, respectively, and $T_{\mathrm{e}}$ for the elastic deformation of the actomyosin network.

\subsection{Myosin kinetics}

The cross-bridge formation between actin and myosin is intimately connected to the intracellular calcium ion concentration. When the muscle cell is activated, ions enter the cytosol via channels in the cell membrane and increase the intracellular ion concentration. This initiates a cascade of events which leads to phosphorylation of myosin and, finally, cross-bridge formation. Following the classical paper by Hai \& Murphy (1988), we assume that smooth muscle myosin can be in four discrete stages: free and unphosphorylated $(\mathrm{M})$, free and phosphorylated $\left(\mathrm{M}_{\mathrm{p}}\right)$, attached and phosphorylated $\left(\mathrm{AM}_{\mathrm{p}}\right)$, attached and dephosphorylated $(\mathrm{AM})$. The transformation between the four stages is given by the kinetic evolution

$$
\left[\begin{array}{c}
\dot{n}_{\mathrm{M}} \\
\dot{n}_{\mathrm{M}_{\mathrm{p}}} \\
\dot{n}_{\mathrm{AM}_{\mathrm{p}}} \\
\dot{n}_{\mathrm{AM}}
\end{array}\right]=\left[\begin{array}{cccc}
-k_{1} & k_{2} & 0 & k_{7} \\
k_{1} & -k_{2}-k_{3} & k_{4} & 0 \\
0 & k_{3} & -k_{4}-k_{5} & k_{6} \\
0 & 0 & k_{5} & -k_{6}-k_{7}
\end{array}\right]\left[\begin{array}{c}
n_{\mathrm{M}} \\
n_{\mathrm{M}_{\mathrm{p}}} \\
n_{\mathrm{AM}_{\mathrm{p}}} \\
n_{\mathrm{AM}}
\end{array}\right]
$$

where $n_{\mathrm{M}}, n_{\mathrm{M}_{\mathrm{p}}}, n_{\mathrm{AM}_{\mathrm{p}}}$, and $n_{\mathrm{AM}}$ denotes the myosin fraction in each of the fours stages, and $k_{1}$ to $k_{7}$ are reaction rates. Since Eq. (5) is given in terms of fractions, the sum of all fractions must also equal unity, i.e., $n_{\mathrm{M}}+n_{\mathrm{M}_{\mathrm{p}}}+n_{\mathrm{AM}_{\mathrm{p}}}+n_{\mathrm{AM}}=1$. Active force is generated when myosin is cycling between the two states $\mathrm{M}$ and $\mathrm{AM}_{\mathrm{p}}$. In addition, Hai \& Murphy (1988) postulated the fourth state AM to account for the low energy expenditure seen in smooth muscles during isometric contraction. This state is often referred to as a latched state where myosin is not cycling, or cycling very slowly.

\subsection{Balance laws}

The balance laws are derived using the principle of virtual power (Gurtin et al., 2010). The principle states that the external and internal power must 
be equal for all admissible virtual velocities of the state variables in quasistatic conditions. Let $\delta \lambda, \delta \varepsilon_{\mathrm{fs}}, \delta \varepsilon_{\mathrm{e}}, \delta \theta$, and $\delta n$ denote the virtual velocities for the state variables $\lambda, \varepsilon_{\mathrm{fs}}, \varepsilon_{\mathrm{e}}, \theta$, and $n$, respectively. The $\delta$ symbol denotes a virtual variation of the variable and the virtual velocities, therefore, play the same role as test functions in finite elements. Note that $\delta \varepsilon_{\mathrm{e}}$ is not arbitrary by Eq. (3), it is merely introduced for convenience. Hence, the external virtual power $\hat{\mathscr{P}}_{\text {ext }}$ per unit referential length is defined as

$$
\hat{\mathscr{P}}_{\mathrm{ext}}=t \delta \lambda+m \delta \theta+t_{n} \delta n,
$$

where $t$ is the total stress, and $m$ and $t_{n}$ are external thermodynamic forces associated with filament sliding and length adaptation, respectively. In addition, the internal virtual power $\hat{\mathscr{P}}_{\text {int }}$ is defined as

$$
\hat{\mathscr{P}}_{\text {int }}=n T_{\mathrm{e}} \delta \varepsilon_{\mathrm{e}}+n T_{\mathrm{AM}_{\mathrm{p}}} \delta w+n T_{\mathrm{AM}} \delta \varepsilon_{\mathrm{fs}}+T_{n} \delta n,
$$

where the internal thermodynamic force $T_{\mathrm{e}}$ is associated with the elastic deformation of the actomyosin, $T_{\mathrm{AM}_{\mathrm{p}}}$ and $T_{\mathrm{AM}}$ are connected to the cycling and non-cycling cross-bridges, respectively, see Fig 2 . The thermodynamic force $T_{n}$ is the internal thermodynamic force associated with length adaptation. The factor $n$ in Eq. (7) appears because the number of contractile units changes during the length adaptation process, see Fig. 1. Equating the internal and external virtual powers and using the arbitrariness of the virtual velocities together with Eqs. (3) and (4), we obtain the balance laws

$$
t=T_{\mathrm{e}} \quad \text { and } \quad T_{\mathrm{e}}=T_{\mathrm{AM}_{\mathrm{p}}}+T_{\mathrm{AM}},
$$

for the mechanical equilibrium, and

$$
\frac{m L_{0}}{r}=n T_{\mathrm{AM}_{\mathrm{p}}} \quad \text { and } \quad t_{n}=T_{n},
$$

associated with contractions and remodelling, respectively.

From the balance laws above, we note that the total stress $t$ is unaffected by remodelling, as is expected for a serial arrangement of the contractile units. In contrast, the external power supplied for active force generation becomes proportional to the number of contractile units by Eq. $(9)^{1}$. The same holds for the isotonic contraction velocity which is evident from Eq. (3) since $\dot{\varepsilon}_{\mathrm{e}}$ is zero for a constant external load. These model characteristics are in perfect agreement with the experimental observations listed in Sect. 1.

\subsection{Constitutive equations}

Consider smooth muscle contraction to be athermal. In this case, constitutive equations can be derived by using the dissipation inequality (Gurtin et al., 2010). The inequality states that the time derivative of the free energy 
$\psi$ must be less or equal to the internal power $\mathscr{P}_{\text {int }}$ for all evolutions of the state variables, i.e.

$$
\dot{\psi} \leq \mathscr{P}_{\text {int }},
$$

where the internal power is obtained by replacing the virtual velocities in Eqs. (7) for their real counterparts. The free energy is modified from Stålhand et al. (2008), and taken to be

$$
\psi=f\left(\varepsilon_{\mathrm{fs}}, n\right) \psi^{\mathrm{e}}\left(\varepsilon_{\mathrm{e}}, \boldsymbol{n}\right),
$$

where $\psi^{\mathrm{e}}$ is the strain-energy function for the actomyosin network, $f \in[0,1]$ is a function describing the filament overlap, and $\boldsymbol{n}=\left(n_{\mathrm{M}}, n_{\mathrm{M}_{\mathrm{p}}}, n_{\mathrm{AM}_{\mathrm{p}}}, n_{\mathrm{AM}}\right)$ is a vector of the myosin state fractions. Substituting Eqs. (7) and (11) into (10) and using (3) gives after rearrangement of terms

$$
\begin{aligned}
& \left(T_{\mathrm{e}}-f \frac{\partial \psi^{\mathrm{e}}}{\partial \varepsilon_{\mathrm{e}}}\right) \dot{\lambda}+\left(-T_{\mathrm{e}}+T_{\mathrm{AM}}+f \frac{\partial \psi^{\mathrm{e}}}{\partial \varepsilon_{\mathrm{e}}}-\frac{\partial f}{\partial \varepsilon_{\mathrm{fs}}} \psi^{\mathrm{e}}\right) n \dot{\varepsilon}_{\mathrm{fs}} \\
& +n T_{\mathrm{AM}_{\mathrm{p}}} w+\left(T_{n}-\frac{\partial}{\partial n}\left(f \psi^{\mathrm{e}}\right)\right) \dot{n} \geq 0 .
\end{aligned}
$$

Equation (12) must hold for all evolutions of the state variables. To that end, assume the deformation of the actomyosin network to be elastic and take

$$
t=T_{\mathrm{e}}=f \frac{\partial \psi^{\mathrm{e}}}{\partial \varepsilon_{\mathrm{e}}},
$$

where Eq. $(8)^{1}$ has been used.

The active force generated by the friction clutch arises from the third term in Eq. (12). Since $n>0$, choose

$$
T_{\mathrm{AM}_{\mathrm{p}}}= \begin{cases}0, & \text { if } w<0, \\ \kappa n_{\mathrm{AM}_{\mathrm{p}}} f w, & \text { if } w \geq 0,\end{cases}
$$

where $\kappa>0$. For this particular choice of $T_{\mathrm{AM}_{\mathrm{p}}}$ we note the following. First, $T_{\mathrm{AM}_{\mathrm{p}}}$ is linear in the slip velocity $w$ and, thus, consistent with the mechanics of a friction clutch. Second, the ensemble of cycling cross-bridges generate a force dependent on the fraction of attached and phosphorylated myosin, i.e., $n_{\mathrm{AM}_{\mathrm{p}}}$, and the filament overlap. Finally, the contractile element only generates contractile force and $T_{\mathrm{AM}_{\mathrm{p}}}$ is set to zero whenever $w<0$ as it would otherwise be contradictory.

Having defined $T_{\mathrm{AM}_{\mathrm{p}}}$, we turn our attention to the evolution laws for $\varepsilon_{\mathrm{fs}}$ and $n$. To obtain an evolution law for $\varepsilon_{\mathrm{fs}}$, we substitute Eq. $(8)^{2}$ into the second term of Eq. (12) and take

$$
-T_{\mathrm{AM}_{\mathrm{p}}}+f \frac{\partial \psi^{\mathrm{e}}}{\partial \varepsilon_{\mathrm{e}}}-\frac{\partial f}{\partial \varepsilon_{\mathrm{fs}}} \psi^{\mathrm{e}}=c_{\mathrm{fs}} \dot{\varepsilon}_{\mathrm{fs}},
$$


where $c_{\mathrm{fs}}>0$ is a constant.

To derive an evolution for the adaptation variable $n$, we take the fourth term of Eq. (12) equal to

$$
T_{n}-\frac{\partial}{\partial n}\left(f \psi^{\mathrm{e}}\right)=g_{n} \dot{n},
$$

where $g_{n} \geq 0$.

The mechanisms governing length adaptation are currently not well understood, as mentioned in Sect. 1. It has, however, been suggested that structural malleability of the actomyosin network is an essential ingredient and that filament polymerisation plays a central role (Bossé et al., 2008; van den Akker et al., 2010). It has also been shown that phosphorylation of regulatory myosin light chains promotes myosin filament polymerisation associated with the actomyosin network (Pratusevich et al., 1995; Kuo et al., 2003). With the latter in mind, $g_{n}$ is taken to be

$$
1 / g_{n}=c_{n}\left(n_{\mathrm{M}_{\mathrm{p}}}+n_{\mathrm{AM}_{\mathrm{p}}}\right),
$$

where $c_{n}>0$ is a constant and $n_{\mathrm{M}_{\mathrm{p}}}+n_{\mathrm{AM}_{\mathrm{p}}}$ is the total fraction of phosphorylated myosin. Furthermore, it has also been suggested that the length adaptation tries to achieve a maximum filament overlap at the current length (Tuna et al., 2011). To this end, we first introduce the bell-shaped filament overlap function

$$
f=\exp \left[\left(\varepsilon_{\mathrm{fs}}-n \mu\right)^{2} / 2 \gamma\right],
$$

where $\gamma$ is a constant and $\mu$ is the active stretch at which the filaments are fully overlapping in the reference state, i.e. when $n=1$. Next, we suggest the following thermodynamic force $T_{n}$, driving remodelling, i.e.

$$
T_{n}=\operatorname{erf}\left[\beta\left(\varepsilon_{\mathrm{fs}}-n \mu\right)\right]+\frac{\partial}{\partial n}\left(f \psi^{\mathrm{e}}\right),
$$

where erf signifies the error function and $\beta>0$ determines the sensitivity of $T_{n}$ with respect to $\varepsilon_{\mathrm{fs}}-n \mu$. This choice makes $\dot{n}<0$ in Eq. (16) whenever $\varepsilon_{\mathrm{fs}}<n \mu$. The adaptation will, therefore, reduce the number of contractile units in series and shift the optimal filament overlap $n \mu$ towards lower values. A completely analogous argument apply if $\varepsilon_{\mathrm{fs}}>n \mu$, but $n \mu$ is now shifted towards higher values.

\section{Parameter identification}

Most of the parameters in the presented model can be obtained from standard force-length and force-velocity experiments. In this section, we will show how this is done. As an example, we will specialise the model to the 

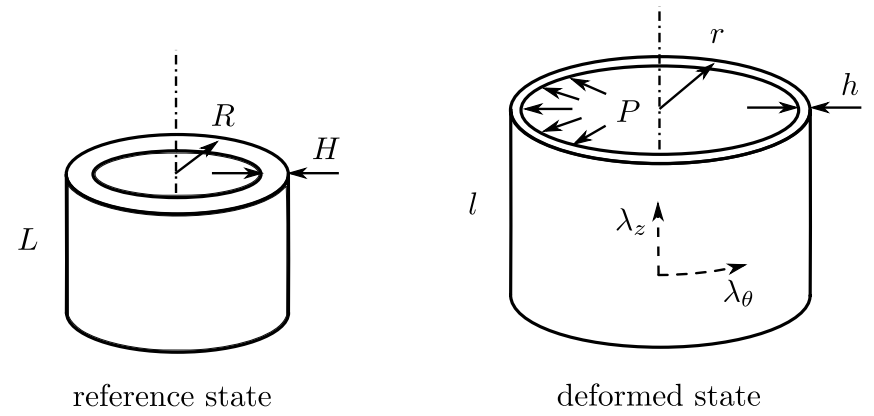

Figure 3: Schematic illustration of the reference and deformed states for the cheek pouch arteriole assumed to be a tube with length $L$, radius $R$, and wall thickness $H$ in the reference state. When subjected to a luminal pressure $P$ the tube has length $l$, radius $r$, and wall thickness $h$. The circumferential stretch $\lambda_{\theta}$ and the axial stretch $\lambda_{z}$ are also indicated.

hamster cheek pouch arteriole using data from Davis \& Gore (1989) and Davis \& Davidson (2002). First, we consider a section of an arteriole in quasi-static conditions. Assume that its stress-free reference state can be approximated by a thin-walled cylinder with radius $R$, wall thickness $H$, and length $L$, see Fig. 3. Let the cylinder be subjected to a luminal pressure $P$ such that it deforms into a cylinder with radius $r$, wall thickness $h$, and length $l$. The circumferential and axial stretches are then given by

$$
\lambda_{\theta}=\frac{r}{R}, \quad \lambda_{z}=\frac{l}{L},
$$

respectively. The radial stretch $\lambda_{r}$ is not an independent quantity since arterial walls are assumed to behave incompressible so that the deformation must satisfy

$$
\lambda_{\theta} \lambda_{z} \lambda_{r}=1 .
$$

For a shear stress free inflation, the Cauchy stresses are given by (Holzapfel, 2000)

$$
\sigma_{i}=-p+\lambda_{i} \frac{\partial \psi}{\partial \lambda_{i}}, \quad i=\theta, z, r,
$$

where the subscripts $\theta, z$, and $r$ indicate the circumferential, axial, and radial direction, respectively. The first term on the right-hand side is an unknown reaction stress (hydrostatic pressure $p$ ) arising from the incompressibility condition while the second term is the elastic stress.

The arteriolar wall can be viewed as a composite material comprising an elastic extracellular matrix of collagen and elastin in which smooth muscle 
cells are embedded in the circumferential direction (Walmsley et al., 1982). The extracellular matrix is not accounted for by the free energy (11) but it can be included by adding the term $\psi^{\mathrm{p}}=\psi^{\mathrm{P}}\left(\lambda_{\theta}, \lambda_{z}, \lambda_{r}\right)$ to the right-hand side. Substituting the amended free energy $\psi=\psi^{\mathrm{p}}+f \psi^{\mathrm{e}}$ into (22) gives

$$
\sigma_{\theta}=-p+\lambda_{\theta} \frac{\partial \psi^{\mathrm{p}}}{\partial \lambda_{\theta}}+\lambda_{\theta} f \frac{\partial \psi^{\mathrm{e}}}{\partial \varepsilon_{\mathrm{e}}}, \quad \sigma_{z}=-p+\lambda_{z} \frac{\partial \psi^{\mathrm{p}}}{\partial \lambda_{z}}, \quad \sigma_{r}=-p+\lambda_{r} \frac{\partial \psi^{\mathrm{p}}}{\partial \lambda_{r}} .
$$

The unknown reaction stress $p$ can be determined by using the fact that the radial stress in a thin walled cylinder is negligible compared to the axial and circumferential stresses. Setting $\sigma_{r}=0$ in Eq. $(23)^{3}$ gives $-p=-\lambda_{r} \partial \psi^{\mathrm{p}} / \partial \lambda_{r}$.

Let the strain energy for the extracellular passive material be

$$
\psi^{\mathrm{p}}=\frac{c_{0}}{2}\left(\lambda_{\theta}^{2}+\lambda_{z}^{2}+\lambda_{r}^{2}\right)+\frac{c_{1}}{2 c_{2}}\left(\exp \left[c_{2}\left(\lambda_{\theta}^{2}-1\right)^{2}\right]-1\right),
$$

where $c_{0}, c_{1}$, and $c_{2}$ are material constants (Holzapfel et al., 2000). To simplify the analysis, collagen fibres are assumed to be oriented in the circumferential direction in Eq. (24). Following Murtada et al. (2010), we take the strain energy for the actomyosin network to be

$$
\psi^{\mathrm{e}}=\frac{K_{\mathrm{e}}}{2}\left(n_{\mathrm{AM}_{\mathrm{p}}}+n_{\mathrm{AM}}\right) \varepsilon_{\mathrm{e}}^{2},
$$

where $K_{\mathrm{e}}$ is a stiffness. The term $n_{\mathrm{AM}_{\mathrm{p}}}+n_{\mathrm{AM}}$ is introduced because elastic energy can only be stored in cross-bridges attached to actin.

The specimen in Davis \& Gore (1989) and Davis \& Davidson (2002) are held at a fixed length during the experiments and $l=L$ which gives $\lambda_{z}=1$ by Eq. $(20)^{2}$. Substituting $\lambda_{z}$ into Eq. $(23)^{1}$ together with Eqs. (24) and (25) gives the circumferential Cauchy stress $\sigma_{\theta}$. The fitting of the model to experimental data in Davis \& Gore (1989) and Davis \& Davidson (2002) is greatly simplified if the stress is given in terms of the first Piola-Kirchhoff stress $t_{\theta}$ rather than the Cauchy stress. Using the connection (Holzapfel, 2000)

$$
\sigma_{\theta}=\lambda_{\theta} t_{\theta}
$$

the first Piola-Kirchhoff stress can be computed by means of Eqs. (18) and (23)-(25). The result reads

$$
\begin{aligned}
t_{\theta}= & c_{0}\left(\lambda_{\theta}-\frac{1}{\lambda_{\theta}^{3}}\right)+2 c_{1} \lambda_{\theta}\left(\lambda_{\theta}^{2}-1\right) \exp \left[c_{2}\left(\lambda_{\theta}^{2}-1\right)^{2}\right] \\
& +K_{\mathrm{e}}\left(n_{\mathrm{AM}_{\mathrm{p}}}+n_{\mathrm{AM}}\right) \exp \left[\left(\varepsilon_{\mathrm{fs}}-n \mu\right)^{2} / 2 \gamma^{2}\right] \varepsilon_{\mathrm{e}} .
\end{aligned}
$$

The circumferential stress in Davis \& Gore (1989) is computed from Laplace's law $\sigma_{\theta}=\operatorname{Pr} / h$. The deformed thickness $h$ can be replaced by 
the referential thickness $H$ using the connection $H=\lambda_{\theta} \lambda_{z} h$ valid for an incompressible tube. Furthermore, substituting Eq. (26) together with $\lambda_{z}=$ 1 into Laplace's law we obtain

$$
t_{\theta}=\frac{P r}{H} \text {. }
$$

The nominator in Eq. (28) is the wall tension and is given (Davis \& Gore, 1989; Davis \& Davidson, 2002). To obtain $t_{\theta}$, we need only to divide the tensile value by the referential wall thickness which is taken to be $H=$ $5.4 \mu \mathrm{m}$, from Table 1 in Davis \& Gore (1989).

The material constants in the passive strain energy (24) are obtained by setting $K_{\mathrm{e}}=0$, in Eq. (27), and fitting $t_{\theta}$ to the passive stress-radius graph in Fig. 3 of Davis \& Gore (1989). Towards this end, the circumferential stretch must be computed from Eq. $(20)^{1}$ and for this we need the referential radius $R$. It is taken to be $21 \mu \mathrm{m}$ which corresponds to the radius at zero pressure in the same graph. The results from the parameter identification is presented in Table 1 and Fig. 4 .

\begin{tabular}{|c|c|c|c|}
\hline \multicolumn{2}{|c|}{ Arteriolar geometry } & \multicolumn{2}{|c|}{ Active material } \\
\hline$R(\mu \mathrm{m})$ & $21^{\dagger}$ & $K_{\mathrm{e}}(\mathrm{kPa})$ & 24370 \\
\hline$H(\mu \mathrm{m})$ & $5.4^{\dagger}$ & $\mu(-)$ & 0.712 \\
\hline$\lambda_{\mathrm{z}}(-)$ & 1.0 & $\gamma(-)$ & 0.5 \\
\hline \multicolumn{2}{|c|}{ Experimental protocol } & $\kappa(\mathrm{kPa} / \mathrm{s})$ & 8220 \\
\hline$t_{\theta, \text { act }}^{\max }(\mathrm{kPa})$ & $190^{\dagger}$ & $c_{\mathrm{fs}}(\mathrm{kPa} / \mathrm{s})$ & 1843 \\
\hline$\lambda_{\theta}^{\max }(-)$ & $1.72^{\dagger}$ & \multicolumn{2}{|c|}{ Myosin kinetics } \\
\hline$v(1 / \mathrm{s})$ & $0.059^{\ddagger}$ & $k_{1}^{\text {ref }}(1 / \mathrm{s})$ & $6.50^{*}$ \\
\hline \multicolumn{2}{|c|}{ Passive material } & $k_{2}, k_{5}(1 / \mathrm{s})$ & $9.72^{*}$ \\
\hline$c_{0}(\mathrm{kPa})$ & 9.914 & $k_{3}(1 / \mathrm{s})$ & $4.00^{*}$ \\
\hline$c_{1}(\mathrm{kPa})$ & 0.102 & $k_{4}(1 / \mathrm{s})$ & $0.05^{*}$ \\
\hline$c_{2}(-)$ & 0.370 & $k_{7}(1 / \mathrm{s})$ & $0.02^{*}$ \\
\hline
\end{tabular}

Table 1: Parameters for the cheek pouch arteriole model. Superscripts indicate that the value is taken from the literature according to: $(\dagger)$ Davis \& Gore (1989), ( $\ddagger$ ) Davis \& Davidson (2002), and (*) Murtada et al. (2012).

To find the model parameters for the active part of the model, we first define the active Cauchy stress by subtracting the passive response $-p+$ $\lambda_{\theta} \partial \psi^{\mathrm{p}} / \partial \lambda_{\theta}$ from Eq. $(23)^{1}$. Next, substitute Eqs. (18) and (25) into the active Cauchy stress and use the connection (26) to obtain the active first Piola-Kirchhoff stress

$$
t_{\theta, \text { act }}=K_{\mathrm{e}}\left(n_{\mathrm{AM}_{\mathrm{p}}}+n_{\mathrm{AM}}\right) \exp \left[\left(\varepsilon_{\mathrm{fs}}-n \mu\right)^{2} / 2 \gamma^{2}\right] \varepsilon_{\mathrm{e}} .
$$

This is a bell-shaped curve with a maximum $t_{\theta \text {,act }}^{\max }$. The maximum stress and its corresponding stretch $\lambda_{\theta}^{\max }$ are easy to read off from the active stressradius graph documented in Davis \& Gore (1989). The filament overlap is 

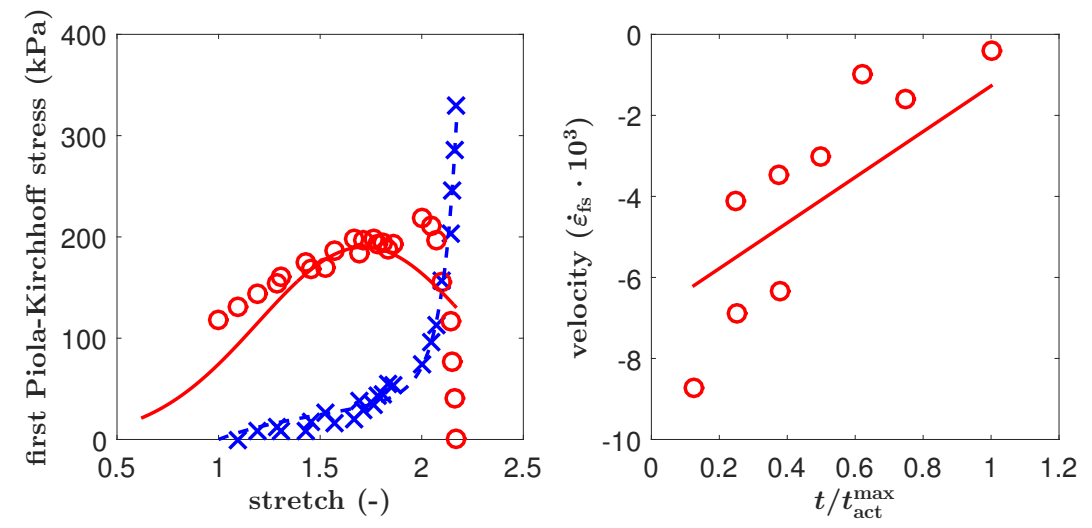

Figure 4: Stress-stretch response for a smooth muscle (left panel). Crosses indicate the passive response while circles indicate the active response. The dashed and solid curves are the passive and active model responses, respectively. Data are taken from Davis \& Gore (1989). Stress-velocity response from a quick-release experiment (right panel). Circles indicate the measured response while the solid line is the model response. Data are taken from Davis \& Davidson (2002).

maximised at this stretch and $\varepsilon_{\mathrm{fs}}=n \mu$, by Eq. (18). The maximum active stress can, therefore, be simplified to

$$
t_{\theta, \text { act }}^{\max }=K_{\mathrm{e}}\left(n_{\mathrm{AM}_{\mathrm{p}}}+n_{\mathrm{AM}}\right) \varepsilon_{\mathrm{e}}^{\max },
$$

where, by Eq. (2),

$$
\varepsilon_{\mathrm{e}}^{\max }=\frac{\lambda_{\theta}^{\max }}{n}-\mu n-1 .
$$

Note that when parameters for the active part of the model are identified, it is assumed that the force-length and force-velocity experiments are conducted in the reference state and $n=1$.

The actomyosin network stiffness $K_{\mathrm{e}}$ can be identified from a quickrelease experiment. Assuming the passive stress to be negligible, the instantaneous shortening following a quick release is only attributed to the actomyosin network, symbolised by the serial spring in Fig. 2. Since the actomyosin network is assumed to be linear, the following must hold for a quick release from the maximum active stress:

$$
t_{\theta, \text { act }}-t_{\theta, \text { act }}^{\max }=K_{\mathrm{e}}\left(n_{\mathrm{AM}_{\mathrm{p}}}+n_{\mathrm{AM}}\right)\left(\varepsilon_{\mathrm{e}}-\varepsilon_{\mathrm{e}}^{\max }\right) .
$$

The filament displacement $\varepsilon_{\mathrm{fs}}$ and $n$ do not change over the instantaneous shortening following a quick release and by Eq. (2), $\varepsilon_{\mathrm{e}}-\varepsilon_{\mathrm{e}}^{\max }=\lambda_{\theta}-\lambda_{\theta}^{\max }$ since $n$ is assumed to be unity. The fraction of attached myosin $n_{\mathrm{AM}_{\mathrm{p}}}+n_{\mathrm{AM}}$ 
can be computed from Eq. (5) using the rate constants, as documented in Murtada et al. (2012). The rate constants $k_{1}$ and $k_{6}$ associated with myosin phosphorylation are computed using Eq. (1) and the stationary intracellular calcium ion level in Fig. 6 in the same reference. Once the stress and stretch differences are known, the stiffness $K_{\mathrm{e}}$ is computed from Eq. (32). Next, with $t_{\theta, \text { act }}^{\max }, \lambda_{\theta}^{\max }, K_{\mathrm{e}}, n_{\mathrm{AM}_{\mathrm{p}}}$, and $n_{\mathrm{AM}}$ in hand, the values for $\varepsilon_{\mathrm{e}}^{\max }$ and $\mu$ are computed from Eqs. (30) and (31), respectively.

The last parameter which describes the active stress curve is $\gamma$ in Eq. (18). It defines the width of the active stress-radius curve and can be obtained by fitting Eq. (29) to the corresponding curve in Davis \& Gore (1989), see Fig. 4.

The clutch velocity $v$ associated with active force generation can also be computed. To that end, start by noting that the maximum contraction velocity generated by the clutch is $\dot{\varepsilon}_{\mathrm{fs}}=-v$. At this velocity the driving force $T_{\mathrm{AM}_{\mathrm{p}}}$ becomes zero by Eq. (14). The maximum contraction velocity from quick-release experiments is reported to be $3.05 \mu \mathrm{m} / \mathrm{s}$, see Table 2 in Davis \& Davidson (2002). Dividing this value by the reference length $2 \pi R$, the normalised contraction velocity can be computed to be $v=0.059 \mathrm{~s}^{-1}$. Next, assume $-v<\dot{\varepsilon}_{\mathrm{fs}} \leq 0$, i.e., a contracting muscle, and substitute Eq. $(14)^{2}$ into (15) followed by a rearrangement of the terms using (4), we obtain

$$
\left(f n_{\mathrm{AM}_{\mathrm{p}}} \kappa+c_{\mathrm{fs}}\right) \dot{\varepsilon}_{\mathrm{fs}}+f n_{\mathrm{AM}_{\mathrm{p}}} \kappa v=f \frac{\partial \psi^{\mathrm{e}}}{\partial \varepsilon_{\mathrm{e}}}-\frac{\partial f}{\partial \varepsilon_{\mathrm{fs}}} \psi^{\mathrm{e}} .
$$

The parameters $\kappa$ and $c_{\mathrm{fs}}$ in Eqs. (14) and (15), respectively, can be computed in the following way: if the specimen is held fixed at $\lambda_{\theta}^{\max }$, then $\dot{\varepsilon}_{\mathrm{fs}}=0$ and $f=1$, and $\partial f / \partial \varepsilon_{\mathrm{fs}}=0$ by Eq. (18). Substituted into (33) together with Eq. (25) and using (30) gives

$$
n_{\mathrm{AM}_{\mathrm{p}}} \kappa v=t_{\theta, \text { act }}^{\max },
$$

from which $\kappa$ can be solved. Finally, $c_{\mathrm{fs}}$ can be obtained by fitting Eq. (33) to the force-velocity data in Davis \& Davidson (2002), see Fig. 4 therein.

This leaves us with only two unknown parameters which cannot be identified from classical muscle experiments, namely, $c_{n}$ and $\beta$. These parameters describe the length adaptation process and require dedicated experiments. Since no such data are available in the literature, to the best of the authors' knowledge, we simulate a typical experimental protocol and perform a parameter study for $c_{n}$ and $\beta$. The model response is then compared to similar experiments.

\section{Length adaptation in uniaxial tension}

To study the behaviour of the model, we simulate a typical experimental protocol for studying length adaptation. In the simulation, a segment of 
hamster cheek pouch arteriole is subjected to sustained uniaxial tension along the circumferential direction. The specimen is initially set to the optimal force generating stretch $\lambda_{\theta}^{\max }$ and activated. It is then allowed to isometrically contract stepwise to $0.9,0.8,0.7$, and 0.6 times the optimal stretch. The specimen is held for 3600 seconds at each length, see Fig. 5. The myosin is assumed to be in a length adapted equilibrium state initially, and the corresponding myosin fractions are computed by solving Eq. (5) for $\dot{n}_{\mathrm{M}}=\dot{n}_{\mathrm{M}_{\mathrm{p}}}=\dot{n}_{\mathrm{AM}_{\mathrm{p}}}=\dot{n}_{\mathrm{AM}}=0$ subject to $n_{\mathrm{M}}+n_{\mathrm{M}_{\mathrm{p}}}+n_{\mathrm{AM}_{\mathrm{p}}}+n_{\mathrm{AM}}=1$. Since the specimen is assumed to be in a length adapted state initially, $n$ is taken to be unity and $\varepsilon_{\mathrm{fs}}$ is set to $\mu$ at the start of the simulation.

The time-dependent problem is now simulated by simultaneously solving Eqs. (5), (15), and (16). The stress and filament overlap are computed using Eqs. (27) and (18), respectively. The model parameters $\beta$ and $c_{n}$ cannot be identified from standard force-length and force-velocity experiments, and the model response is computed for $\beta=0.2,0.5,1.0,2.0,5.0,10.0$ and $c_{n}=$ $0.001,0.002,0.005,0.01 \mathrm{Js} / \mathrm{m}^{3}$. All other parameters are taken from Table 1.

A representative model response for $\beta=1.0$ and $c_{n}=0.002$ is shown in Fig. 5. The step-wise change in the muscle length (top left panel) causes a rapid change in the filament overlap $\varepsilon_{\mathrm{fs}}$ (top right panel) away from its maximum. The imbalance between $\varepsilon_{\mathrm{fs}}$ and $n \mu$ in Eq. (19) causes a remodelling of the number of contractile units $n$ (bottom left panel) which returns the filament overlap towards its maximum (bottom right panel). The corresponding active stress is shown in Fig. 6. The filament displacement caused by the step-wise length change reduces the active force initially, but the isometric stress gradually recovers as length adaptation sets in.

The model response to the parameters $\beta$ and $c_{n}$ is shown in Fig. 7. Each point in the graph represents the mean time $t_{1 / 2}$ for $n$ to reach half of its new steady-state value for the four consecutive length steps in Fig. 5. The whiskers indicate the variation in the half time between the four consecutive length steps (one standard deviation). Note that for $\beta$ approximately larger than 2 , the variation is too small for the whiskers to be visible.From the graph, it is readily concluded that an increase in both $\beta$ and $c_{n}$ lead to a faster remodelling process. For low values of $c_{n}$ and $\beta$, the length adaptation was not completed after 60 mins and these results are excluded from Fig. 7 .

The stress recovery in $t$ after a length step can approximately be described by the exponential function $a[1-\exp (-b t)]$, where $a$ is the active stress immediately after the length step and $b$ is a constant. When half the stress is recovered, the time constant $b$ may be computed as $b=\ln 2 / \tau$, where $\tau$ is the half-time indicated by a ring in the insert of Fig. 6 . For $c_{n}=0.002$ and $\beta=1$, the half-time $\tau$ is approximately $2.65 \mathrm{~min}$ and $b=0.26$. This number is on the same order of magnitude as the time constant 0.145 for sheep pulmonary arteries, as reported by Syyong et al. (2008). 

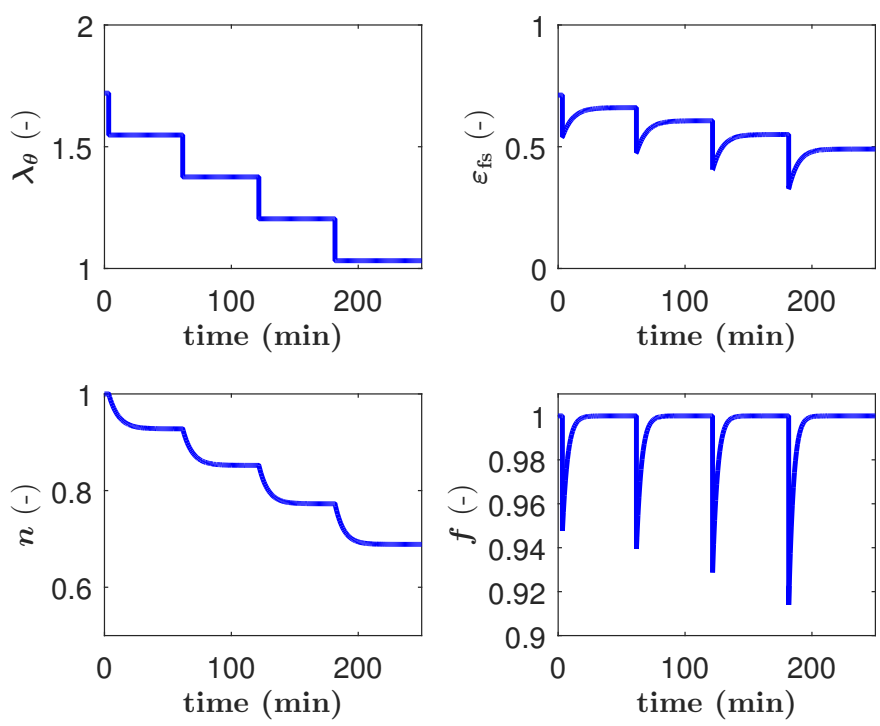

Figure 5: Representative response for the simulated length adaptation experiment. The circumferential stretch $\lambda_{\theta}$ during the stepwise contractions (top left) and the response in the filament sliding strain $\varepsilon_{\mathrm{fs}}$ (top right), the fraction of contractile units $n$ (bottom left), and the filament overlap $f$ (bottom right). The remodelling parameters are $\beta=1.0$ and $c_{n}=0.002$.

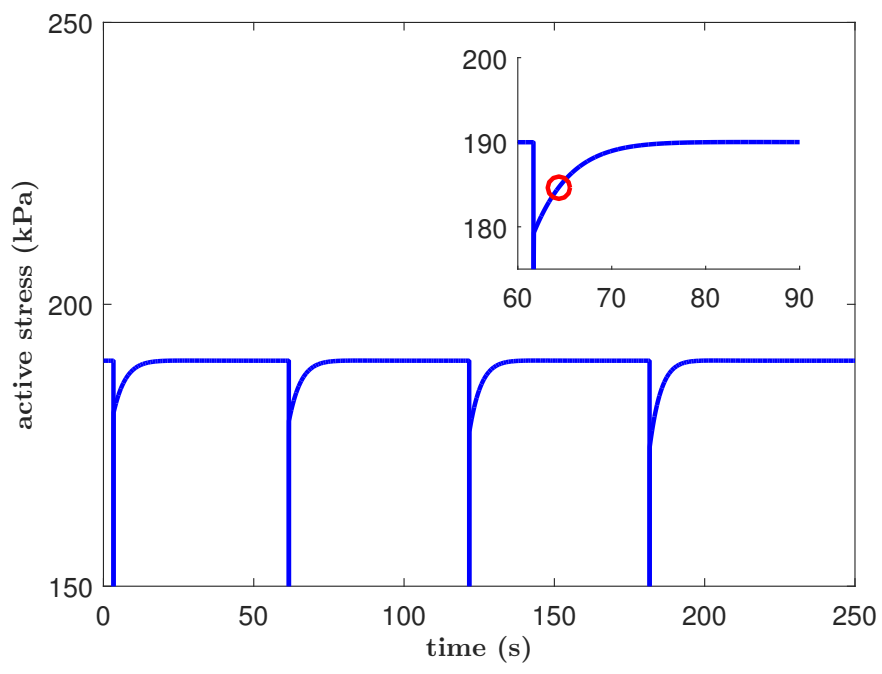

Figure 6: Representative active stress response for the simulated length adaptation experiment. The inserted picture is a magnification of the transient stress response in the second step when the specimen length is reduced from 0.9 to $0.8 L_{0}$. The red circle indicates the time when the isometric stress has recovered half of its value and the vertical lines at each length step are the transient response from the serial spring. The remodelling parameters are $\beta=1.0$ and $c_{n}=0.002$. 


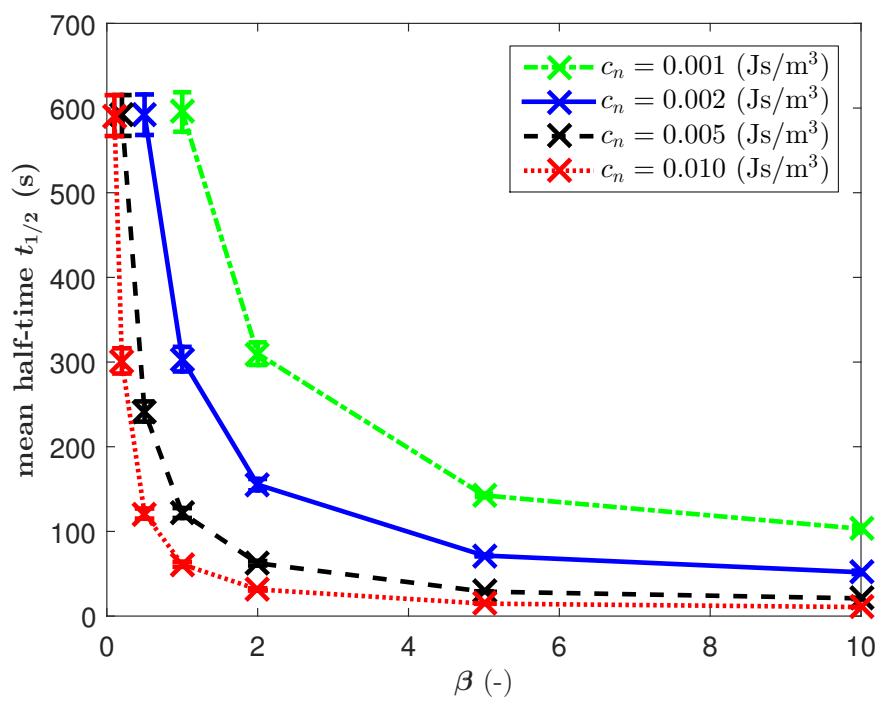

Figure 7: Mean time $t_{1 / 2}$ for the length remodelling variable $n$ to reach half of its new steady state value after a length step as a function of the constants $c_{n}$ and $\beta$. Crosses indicate the mean value of the four consecutive length steps while whiskers show the variation between the four length steps (one standard deviation). Note that for $\beta$ approximately larger than 2 , the variation is too small for the whiskers to be visible.

\section{Discussion}

The model developed in the present study focuses on length adaptation of the contractile filaments. In reality, it is not only the contractile filaments that adapt in response to a length step; also other cytoskeletal components (Gunst et al., 2003; Herrera et al., 2004) and the extracellular matrix will undergo remodelling. For the time scale considered here, the remodelling of the extracellular matrix is negligible, but cytoskeletal remodelling is likely to take place (Wang et al., 2001). Although not considered in this study, there is a close link between contraction and the rapid formation of actin and myosin filaments beyond cross-bridge cycling (Seow, 2005). For example, actin polymerisation inhibitors blocks the development of full contraction force in resistance (peripheral) vessels (Mehta \& Gunst, 1999). Increasing evidence indicates that actin polymerisation strengthens and stiffens the cytoskeleton whereby the force transmission efficiency increases (MartinezLemus et al., 2008). To the best of the authors' knowledge, data for remodelling of cytoskeletal components other than the contractile filaments have not yet been reported, at least data suitable for continuum mechanical modelling. These components are, therefore, not considered herein.

Figure 4 shows that the model's passive response (dashed curve) captures the behaviour of the experimental data (crosses) well. This cannot be said 
for the active response, however. The model fit to experimental data is good for circumferential stretches in the interval 1.3-1.8. Outside this region there is an increasing discrepancy in the active response between the model and the data. This is particularly noticeable for stretches above 1.8 where the experimental data assume their maximum stress of approximately $230 \mathrm{kPa}$ for the circumferential stretch 2.0, and then rapidly drops to zero. In fact, the measured active stress has a marked negative skew (longer left tail) and is not well described by the bell-shaped function, as provided by Eq. (18). Whether this skewness is physiological or an artefact of the experimental setup is currently not known. We therefore choose to use Eq. (18) since the active response of smooth muscles are usually well described by a bellshaped function, see, e.g., Kamm \& Stull (1985), Murtada et al. (2012), Tuna et al. (2013a) and Murtada et al. (2015). In addition, Eq. (18) has a simple mathematical structure and only a few parameters which is advantageous in the parameter identification.

To identify $K_{\mathrm{e}}$ it was assumed that the passive stress is negligible in an arteriole. This is usually a good approximation for striated muscle where the stretch for maximum force generation is reached before the passive stress becomes significant (Sharifimajd \& Stålhand, 2013). This is not the case for the cheek pouch specimen simulated herein. It reaches its maximum active force at a stretch where the passive material carries a small but significant load, see Fig. 4. Neglecting the influence of the passive material will have an impact on the identification of the actomyosin stiffness $K_{\mathrm{e}}$. Equation (32) assumes that the instantaneous shortening following a quick-release only changes the length of the serial spring in Fig. 2. If the passive material is included in the model by adding a second spring in parallel with the contractile unit, the total load will be distributed between the two springs reducing the load carried by the serial spring. As a consequence, the actomyosin stiffness $K_{\mathrm{e}}$ is overestimated by this assumption.

The evolution law (15) for $\varepsilon_{\mathrm{fs}}$ can be shown to specialise to the Hill equation under certain assumptions, see Sharifimajd \& Stålhand (2013). One of the assumptions is that the quick-release is made from a full filament overlap such that the term $\partial f / \partial \varepsilon_{\mathrm{fs}}=0$ holds. Rather than taking this approach, we chose to compute $\varepsilon_{\mathrm{fs}}$ directly from Eq. (15). A consequence of this choice is that the hyperbolic quick-release data in Fig. 4 is fitted by a linear model response. This indicates that the current model is, probably, best suited for studying small variations in the normalised stress $t / t_{\mathrm{act}}^{\max }$. A better fit could have been obtained by taking the right-hand side of the evolution law equal to a higher-order polynomial, e.g.,

$$
c_{\mathrm{fs}} \dot{\varepsilon}_{\mathrm{fs}}+c_{\mathrm{fs}}^{\prime} \dot{\varepsilon}_{\mathrm{fs}}^{3}+c_{\mathrm{fs}}^{\prime \prime} \dot{\varepsilon}_{\mathrm{fs}}^{5}
$$

where $c_{\mathrm{fs}}, c_{\mathrm{fs}}^{\prime}$, and $c_{\mathrm{fs}}^{\prime \prime}$ are positive constants. This still guarantees a dissipative behaviour, but at the expense of two more constants which must 
be identified. In addition, the parameter identification becomes much more involved since $\dot{\varepsilon}_{\mathrm{fs}}$ cannot be computed directly but must be solved using an iterative scheme. For this reason, we choose to use Eq. (15) in this work.

The model response to the length adaptation parameters $\beta$ and $c_{n}$ can be seen in Figs. 5 to 7. The recovery of the isometric stress in Fig. 6 is very similar to the experiments in, e.g., Pratusevich et al. (1995), Seow (2005), and Wang et al. (2001). The half-time for isometric stress recovery varies depending on the smooth muscle type used. Wang et al. (2001) reported a half-time of 3-5 mins (depending on the length the specimen was adapted to) for rabbit airway smooth muscle while it was approximately 7 mins for rabbit carotid arteries, see Seow (2000). Comparing with Fig. 7, this gives a range for $\beta$ from 0.2 to 4 . In addition, Tuna et al. (2013a) also reported a length adaptation in an in vivo study on rat mesenteric arteries at low flow. The half-time was significantly longer, however, no horizontal shift in the optimal stretch was observed until after 1 day. In contrast, Murtada et al. (2015) studied mice descending aortas under sustained contraction and observed alterations in the magnitude of the bell-shaped stress-stretch curve but no horizontal shift in the optimal stretch. Both the structure and function of the descending aorta and smaller arteries or arterioles differ, however, which may explain the different behaviour.

The thermodynamic force $T_{n}$ in Eq. (19) has two terms. The first term is an error function dependent on the stimulus $\varepsilon_{\mathrm{fs}}-n \mu$. The error function is chosen to make $T_{n}$ saturated as the stimulus tends to plus or minus infinity. The stimulus $\varepsilon_{\mathrm{fs}}-n \mu$ may be seen as a measure of the distance to the optimal filament overlap. The adaptation process is, in reality, most likely dependent on several stimuli, including both chemical and mechanical, which activates different signalling pathways in the cell. Since this model is based on a thermodynamic theory, the former group is not modelled explicitly and the response is solely governed by the mechanical stimulus, i.e., $\varepsilon_{\mathrm{fs}}-n \mu$. The second term in (19) is related to the strain energy stored in the actomyosin network, including cross-bridges. This term is included to cancel the strainenergy derivative in (16) and gives the simple evolution law $T_{n}=g_{n} \dot{n}$.

The length adaptation modelled in this study is based on an assumption that the filament length is constant and contractile units are added or removed in a serial manner. Another way to obtain length adaptation which gives an invariant maximum contractile force and a contraction velocity linear in $n$ is to increase the length of the filaments, see Herrera et al. (2005) and Murtada et al. (2015). To the best of the authors' knowledge, there is no conclusive evidence which assumption is correct. 


\section{Acknowledgments}

This work was supported by the Swedish Research Council (Grant number 621-2009-3099). Their support is hereby gratefully acknowledged.

\section{References}

Bakker, E. N. T. P., Buus, C. L., VanBavel, E., \& Mulvany, M. J. (2004). Activation of resistance arteries with endothelin-1: from vasoconstriction to functional adaptation and remodeling. J. Vasc. Res. 41, 174-182.

Bossé, Y., Sobieszek, A., Paré, P. D., \& Seow, C. Y. (2008). Length adaption of airway smooth muscle. Proc. Am. Thorac. Soc. 5, 62-67.

Davis, M. J., \& Davidson, J. (2002). Force-velocity relationship of myogenically active arterioles. Am. J. Physiol. Heart Circ. Physiol. 282, H165H174.

Davis, M. J., \& Gore, R. W. (1989). Length-tension relationship of vascular smooth muscle in single arterioles. Am. J. Physiol. 256, H630-H640.

Debold, E. P. \& Warshaw,D. M. (2005). Slip sliding away: Load-dependence of velocity generated by skeletal muscle myosin molecules in the laser trap. Biophys. J. 89, L34-L36.

Donovan, G. M. (2013). Modelling airway smooth muscle passive length adaptation via thick filament length distributions. J. Theor. Biol. 333, 102-108.

Gordon, A. M., Huxley, A. F., \& Julian, F. J. (1966). The variation in isometric tension with sarcomere length in vertebrate muscle fibres. J. Physiol. 184, 170-192.

Gunst, S. J., Tang, D. D., \& Opazo Saez, A. (2003). Cytoskeletal remodeling of the airway smooth muscle cell: a mechanism for adaptation to mechanical forces in the lung. Respir. Physiol. Neurobiol. 137, 151-168.

Gurtin, M. E., Fried, E., \& Anand, L. (2010). The Mechanics and Thermodynamics of Continua. New York: Cambridge University Press.

Hai, C. M., \& Murphy, R. A. (1988). Cross-bridge phosphorylation and regulation of latch state in smooth muscle. Am. J. Physiol. 254, C99C106.

Herrera, A. M., Kuo, K.-H., \& Seow, C. Y. (2002). Influence of calcium on myosin thick filament formation in intact airway smooth muscle. Am. J. Physiol. Cell Physiol. 282, C310-C316. 
Herrera, A. M., Martinez, E. C., \& Seow, C. Y. (2004). Electron microscopy study of actin polymerization in airway smooth muscle. Am. J. Physiol. Lung Cell Mol. Physiol. 286, L1161-L1168.

Herrera, A. M., McParland, B. E., Bienkowska, A., Tait, R., Paré, P. D., \& Seow, C. Y. (2005). 'Sarcomeres' of smooth muscle: functional characteristics and ultrastructural evidence. J. Cell Sci. 118, 2381-2392.

Holzapfel, G. A. (2000). Nonlinear Solid Mechanics. A Continuum Approach for Engineering. Wiley \& Sons. Chichester: Wiley \& Sons. Chichester.

Holzapfel, G. A., Gasser, T. C., \& Ogden, R. W. (2000). A new constitutive framework for arterial wall mechanics and a comparative study of material models. J. Elast. 61, 1-48.

Ijpma, G., Al-Jumaily, A. M., Cairns, S. P., \& Sieck, G. C. (2011). Myosin filament polymerization and depolymerization in a model of partial length adaptation in airway smooth muscle. J. Appl. Physiol. 111, 735-742.

Kamm, K. E., \& Stull, J. (1985). Myosin phosphorylation, force, and maximal shortening velocity in neurally stimulated tracheal smooth muscle. Am. J. Phsyiol. Cell Physiol. 249, C238-C247.

Kuo, K.-H., Herrera, A. M., Wang, L., Paré, P. D., Ford, L. E., Stephens, N. L., \& Seow, C. Y. (2003). Structure-function correlation in airway smooth muscle adapted to different lengths. Am. J. Phsyiol. Cell Physiol. 285, C384-C390.

Martinez-Lemus, L. A., Hill, M. A., Bolz, S. S., Pohl, U., \& Meininger, G. A. (2004). Acute mechanoadaption of vascular smooth muscle cells in response to continuous arteriolar vasoconstriction: implications for functional remodeling. FASEB J. 18, 708-710.

Martinez-Lemus, L. A., Hill, M. A., \& Meininger, G. A. (2008). The plastic nature of the vascular wall: a continuum of remodeling events contributing to control of arteriolar diameter and structure. Physiology, 24, 45-57.

Mehta, D., \& Gunst, S. J. (1999). Actin polymerization stimulated by contractile activation regulates force development in canine tracheal smooth muscle. J. Physiol. 519, 829-840.

Murtada, S.-I., Holzapfel, G. A., \& Arner, A. (2012). Experiments and mechanochemical modeling of smooth muscle contraction: significance of filament overlap. J. Theor. Biol. 297, 176-186.

Murtada, S.-I., Holzapfel, G. A., \& Kroon, M. (2010). A calcium-driven mechanochemical model for prediction of force generation in smooth muscle. Biomech. Modeling Mechanobiol. 9, 749-762. 
Murtada, S.-I., Lewin, S., Arner, A., \& Humphrey, J. D. (2015). Adaptation of active tone in the mouse descending thoracic aorta under acute changes in loading. Biomechan. Modeling Mechanobiol. doi:10.1007/s10237-0150711-z.

Pratusevich, V. R., Seow, C. Y., \& Ford, L. E. (1995). Plasticity in canine airway smooth muscle. J. Gen. Physiol. 105, 73-94.

Seow, C. Y. (2000). Response of arterial smooth muscle to length perturbation. J. Appl. Physiol., 89, 2065-2072.

Seow, C. Y. (2005). Myosin filament assembly in an ever-changing myofilament lattice of smooth muscle. Am. J. Physiol. Cell Physiol. 289, C1363-C1368.

Sharifimajd, B., \& Stålhand, J. (2013). A continuum model for skeletal muscle contraction at homogeneous finite deformations. Biomech. Modeling Mechanobiol. 12, 965-973.

Sharifimajd, B., \& Stålhand, J. (2014). A continuum model for excitationcontraction of smooth muscle under finite deformations. J. Theor. Biol. $355,1-9$.

Silveira, P. S., Butler, J. P., \& Fredberg, J. J. (2005). Length adaptation of airway smooth muscle: a stochastic model of cytoskeletal dynamics. J. Appl. Physiol. 99, 2087-2098.

Stålhand, J., Klarbring, A., \& Holzapfel, G. A. (2008). Smooth muscle contraction: mechanochemical formulation for homogeneous finite strains. Prog. Biophys. Molec. Biol. 96, 465-481.

Syyong, H., Cheung, C., Solomon, D., Seow, C. Y., \& Kuo, H. K. (2008). Adaptive response of pulmonary arterial smooth muscle to length change. J. Appl. Physiol. 104, 1014-1020.

Tuna, B. G., Bakker, E. N. T. P., \& VanBavel, E. (2011). Smooth muscle biomechancis and plasticity: relevance for vascular calibre and remodelling. Basic Clin. Pharmacol. Toxocol. 110, 35-41.

Tuna, B. G., Bakker, E. N. T. P., \& VanBavel, E. (2013a). Relation between active and passive biomechanics of small mesenteric arteries during remodeling. J. Biomech. 46, 1420-1426.

Tuna, B. G., Schoorl, M. J. C., Bakker, E. N. T. P., de Vos, J., \& VanBavel, E. (2013b). Smooth muscle contractile plasticity in rat mesenteric small arteries: Sensitivity to specific vasoconstrictors, distension and inflammatory cytokines. J. Vasc. Res. 50, 249-262. 
van den Akker, J., Schoorl, M. J. C., Bakker, E. N. T. P., \& vanBavel, E. (2010). Small artery remodeling: current concepts and questions. J. Vasc. Res. 47, 183-202.

Walmsley, J. G., Gore, R. W., Dacey Jr., R. G., Damon, D. N., \& Duling, B. R. (1982). Quantitative morphology of arterioles from the hamster cheek pouch related to mechanical analysis. Microvasc. Res. 24, 249-271.

Wang, L., Paré, P. D., \& Seow, C. Y. (2001). Selected contribution: effect of chronic passive length change on airway smooth muscle length-tension relationship. J. Appl. Physiol. 90, 734-740. 